\title{
ACM International Conference on Multimedia Retrieval (ICMR): current standing and impact
}

\author{
Michael S. Lew ${ }^{1}$
}

Received: 11 July 2017 / Revised: 21 July 2017 / Accepted: 30 July 2017 / Published online: 7 August 2017

(C) Springer-Verlag London Ltd. 2017

The ACM International Conference on Multimedia Retrieval (ICMR, www.acmicmr.org) was started in 2011 as the flagship meeting covering the dynamic and ubiquitous domain of multimedia search and retrieval. It combined the notable ACM CIVR and MIR conferences and is currently held annually each summer (e.g., www.icmr2018.org is from June 11 to 14 and has CFP due on January 20, 2018).

The growth and pervasiveness of multimedia has been enormous. Already, we have seen the major changes in the way that media, whether it is news or music or video, is being communicated and retrieved. Home multimedia retrieval technology such as Amazon Echo and Google Home has breached the final barrier between multimedia collections and everyday users. Services such as Apple iTunes, Netflix and Amazon Prime supplemented by digital streaming from all major channels have created an environment where finding multimedia information is so prevalent that many governments are passing laws which make Internet access on a similar level of importance and necessity as clean air, health and public transportation. Searching, browsing, recommending and retrieving multimedia are the challenges that billions of people face everyday.

So, how is ICMR doing-how is it ranked internationally and what kind of citation numbers do the papers get?

Based on the best available conference rankings and citation sources, ICMR has become a top tier meeting comparable to the best multimedia conferences and journals in both visibility and citations as explained further below.

Because indexes such as Thomson Reuters do not give impact factors to conferences, we turn to the Google Conference Rankings which are free and publicly available and most importantly can be verified by anyone.

Below is the current standing of ACM ICMR as of July 4, 2017.

Michael S. Lew

mlew@liacs.nl

1 Leiden University, Niels Bohrweg 1, 2333 CA Leiden, The Netherlands 


\begin{tabular}{l} 
Google Scholar \\
Top publications \\
Categories $>$ Engineering \& Computer Science > Multimedia - \\
\hline \\
\hline Publication
\end{tabular}

In comparison with journals and conferences in the general domain of multimedia, ACM ICMR ranks 9th, in a comparable level with prestigious journals and conferences from IEEE and ACM, and ICMR also has had the highest impact factor for a conference in multimedia according to CiteScholar (their index is also based on public Google Scholar citations). On behalf of the ICMR committees, we think it is reasonable to conclude that ICMR is one of the top conferences in the world. Furthermore, publishing in ICMR will give top-tier visibility and citations.

For the historical record, as of July 10, 2017, the three most cited papers in ICMR are the following:

\section{Multi-view face detection using deep convolutional neu- ral networks}

SS Farfade, MJ Saberian, LJ Li

Cited by 113
Getting the look: clothing recognition and segmentation for automatic product suggestions in everyday photos

Y Kalantidis, L Kennedy, LJ Li

Cited by 72

Social event detection using multimodal clustering and integrating supervisory signals

G Petkos, S Papadopoulos, Y Kompatsiaris

Cited by 63

For reference, a screenshot from Google Scholar of the top 10 ICMR papers sorted by citations is given below: 
Multi-view Face Detection Using Deep Convolutional Neural Networks

SS Farfade, MJ Saberian, LJ Li
Proceedings of the 5th ACM on International Conference on Multimedia

Getting the look: clothing recognition and segmentation for automatic product suggestions in everyday photos.

Y Kalantidis, L Kennedy, LJ Li

Social event detection using multimodal clustering and integrating supervisory signals

G Petkos, S Papadopoulos, Y Kompatsiaris

Proceedings of the 2nd ACM International Conference on Multimedia Retrieval, 23

Event-based classification of social media streams

TReuter, P Cimiano

Proceedings of the 2nd ACM International Conference on Multimedia Retrieval, 22

A shape-based approach for leaf classification using multiscaletriangular representation.

$S$ Mouine, I Yahiaoui, A Verroust-Blondet

Recommendations for video event recognition using concept vocabularies.

AH Habibian, KEA van de Sande, CGM Snoek

Event-Driven Semantic Concept Discovery by Exploiting Weakly Tagged Internet Images.

J Chen, Y Cui, G Ye, D Liu, SF Chang

Brand Data Gathering From Live Social Media Streams.

Y Gao, F Wang, HB Luan, TS Chua

Zero-Example Event Search using MultiModal Pseudo Relevance Feedback

L Jiang, T Mitamura, SI Yu, AG Hauptmann

Congratulations to all of the authors, organizers and participants of ICMR for making it a vibrant and high-quality meeting.

This is the Age of Information and ICMR is at the center of it.

Hope to see you at the next ICMR!

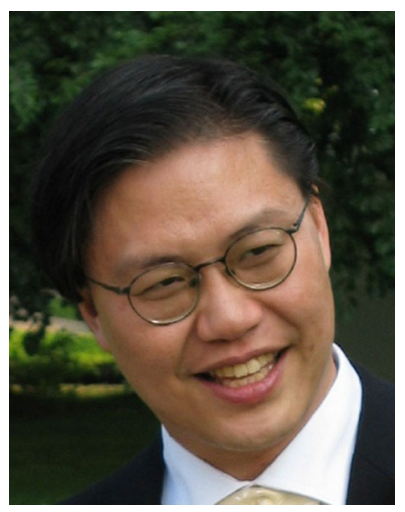

Michael S. Lew

Editor-in-Chief

International Journal of Multimedia Information Retrieval 